\title{
Neuronal entropy depends on the level of alertness in the parkinsonian globus pallidus in vivo
}

\author{
Daniela Sabrina Andres ${ }^{1,2,3}$ *, Daniel Cerquetti ${ }^{2}$, Marcelo Merello ${ }^{2}$ and Ruedi Stoop \\ 1 Institute of Neuroinformatics, ETH Zurich and University of Zurich, Zurich, Switzerland \\ ${ }^{2}$ Movement Disorders Section, Institute for Neurological Research Raul Carrea, Fleni Institute, Buenos Aires, Argentina \\ ${ }^{3}$ Society in Science, The Branco-Weiss Fellowship, ETH Zurich, Zurich, Switzerland
}

Edited by:

Ryuji Kaji, Tokushima University

Hospital, Japan

Reviewed by:

Miguel Coelho, University of Lisbon, Portugal

Andrew P. Duker, University of

Cincinnati, USA

*Correspondence:

Daniela Sabrina Andres, Institute of Neuroinformatics, ETH Zurich and

University of Zurich,

Winterthurerstrasse 190, Zurich 8057,

Switzerland

e-mail:dandres@ini.uzh.ch
A new working hypothesis of Parkinson's disease (PD) proposes to focus on the central role of entropy increase in the basal ganglia $(B G)$ in movement disorders. The conditions necessary for entropy increase in vivo are, however, still not fully described. We recorded the activity of single globus pallidus pars interna neurons during the transition from deep anesthesia to full alertness in relaxed, head-restrained, control, and parkinsonian (6-hydroxydopamine-lesioned group-lesioned) rats. We found that during awakening from anesthesia, the variation of neuronal entropy was significantly higher in the parkinsonian than in the control group. This implies in our view that in PD the entropy of the output neurons of the BG varies dynamically with the input to the network, which is determined by the level of alertness. Therefore, entropy needs to be interpreted as a dynamic, emergent property that characterizes the global state of the BG neuronal network, rather than a static property of parkinsonian neurons themselves. Within the framework of the "entropy hypothesis," this implies the presence of a pathological feedback loop in the parkinsonian BG, where increasing the network input results in a further increase of neuronal entropy and a worsening of akinesia.

Keywords: basal ganglia, Parkinson's disease, entropy, alertness, emergent properties, non-linear

\section{INTRODUCTION}

The measurement of neuronal entropy can be used to characterize the behavior of neural systems (1-4). The concept of entropy was developed in the field of thermodynamics, where it is related to the number of possible states of a system, and can be interpreted as a measure of disorder. Applying this idea to the study of neural systems, neuronal entropy can be associated roughly with the level of disorder present in the discharge of single neurons. From this follows that neuronal entropy shows a negative relation with the predictability of the occurrence of single spikes or, in other words, with the amount of information transmitted by a neuron in the form of temporal patterns. A field where this approach has yielded particularly successful results is Parkinson's disease (PD). During the last years, a growing body of evidence has shown that entropy is increased throughout the parkinsonian basal ganglia (BG) (5-7). Furthermore, a correlation between a decrease in neuronal entropy and anti-parkinsonian therapy was found. In MPTP-treated monkeys, the level of entropy of the internal as well as the external globus pallidum (GPi and GPe, respectively) and the motor thalamus (Th) can be reduced by the application of high frequency deep brain stimulation (DBS) (8). In human patients with $\mathrm{PD}$, an inverse relation between neuronal entropy and the effect of apomorphine has also been reported (9). These results indicate that the clinical effectiveness of antiparkinsonian treatment is correlated with a decrease in neuronal entropy throughout the BG-Th circuit. In this context, it is clear that the evaluation of neuronal entropy is becoming increasingly relevant for the understanding of the pathophysiology of the BG, and might be a key concept in the future for the development of clinical interventions.

A model of BG dysfunctions was recently proposed, which is based on the central role that entropy might play altering the "legibility" of information transmitted through the BG (10). Developing the so called "entropy hypothesis," the authors suggest that the progressive motor impairment in PD might be related to an increasing "illegibility" of neuronal information. The entropy hypothesis is mainly based on two facts: first, anti-parkinsonian treatments decrease the abnormally high entropy in the parkinsonian BG, and second, hyperkinetic conditions such as dystonia are associated with an abnormally low entropy level in the BG (8, $9,11)$. Therefore, hyper- and hypo-kinetic movement disorders seem to be placed at the opposite extremes of a spectrum characterized by the level of entropy of the neuronal discharge of the BG. However, up to now the conditions necessary for the emergence of this phenomenon have not been described. To better understand the changes underlying the increased entropy of the BG in PD, we analyze the variation in the level of entropy in the neuronal discharge of the rat GPi during the transition from deep anesthesia to relaxed, full alertness. Since GPi neurons are essentially output nodes of the BG network, any modification of the information/entropy content in their discharge will be reflected in a necessary disruption of the communication between the BG and the Th/cortex. By studying the GPi during the awakening from anesthesia, we may gain insight into the way that the BG respond to an increasing level of un-specific input in vivo in the healthy vs. the parkinsonian condition. 
Our study is motivated by the application of the surgical treatment of PD, and in particular DBS, a therapy that has been successful in alleviating PD symptoms in selected groups of patients for decades (12-14). The implantation surgery of DBS electrodes takes place usually while the patient is awake, under local anesthesia only $(15,16)$. Under these conditions, microrecording of the neuronal activity along the surgical tract is the gold standard procedure for the identification of the surgical target (17-19). In previous works, we have emphasized the importance of a careful characterization of signals obtained from the patients' own brains, for both research and clinical purposes $(20,21)$. However, the fact that the subjects are alert during the recording makes the interpretation of these data difficult, since measured properties can be influenced by the alertness state as well as the severity of the disease. In the human $\mathrm{BG}$ in patients with $\mathrm{PD}$, contradictory results have been reported between alertness and deep anesthesia (22-25). In animals, on the other hand, the discrimination between the effect of the level of alertness and that of sensory stimuli or motor activity remains technically challenging (26-28). Our experiments were designed to mimic the human surgery in PD patients as much as possible. With the aim of exploring the effect of alertness over the healthy and parkinsonian GPi, all the neuronal recordings were obtained with the animals relaxed, under environmental silence and with their eyes covered, guaranteeing the absence of motor activity, as well as any auditory and visual stimuli at the times of the experiments.

\section{MATERIALS AND METHODS ETHICS STATEMENT}

All animal experiments and procedures were conducted with adherence to the norms of the Basel Declaration (29). The experimental protocol was revised and approved by our local ethics committee CEIB, Buenos Aires, Argentina. All experiments took place at the authorized laboratories of our center, and adherence to the Basel Declaration standards were monitored by our research staff. During the time previous and between experiments, animals were housed in racks with optimal temperature, pressure, and air humidity regulation under an inverted $12 \mathrm{~h}$ light cycle, with water and food available ad libitum. To minimize animal suffering, optimal anesthetic and analgesic medication were used as described below. Euthanasia was conducted using a high dose of meperidine, an opioid suitable for that purpose, guaranteeing the absence of animal suffering during the procedure. In compliance with the 3$\mathrm{R}$ principles, the number of animals used in the experiments was the minimum considered necessary to achieve sound conclusions.

\section{ANIMAL MODEL}

Adult male and female Sprague-Dawley rats weighing 250-350 g were randomly divided in three groups: 6-hydroxydopaminelesioned group (6OHDA), Sham-lesioned group, and not-lesioned group. The choice of the three experimental groups obeyed the following rationale: the 6OHDA-lesioned group modeled the parkinsonian case and the healthy group served as a general control group, while the comparison of these two groups with the Sham-lesioned group guaranteed that the behavioral deficits in the parkinsonian model were due to the chemical action of the toxin and not to any other effect product of the surgical procedure itself. Animals within the 6OHDA group were lesioned unilaterally following the partial-lesion model originally described by Sauer and Oertel (30). A total dose of $20 \mu \mathrm{g}$ of 6OHDA diluted in $4 \mu \mathrm{l}$ of saline solution supplemented with $0.2 \mathrm{mg} / \mathrm{ml}$ of ascorbic acid was injected at a rate of $1 \mu \mathrm{l} / \mathrm{min}$ in the striatum (Str - coordinates: anterio-posterior, +1.0; lateral, +3.0 ; depth, -4.5$)$ using a Hamilton microsyringe. After waiting $5 \mathrm{~min}$ from the time, the injection was completed, the syringe was withdrawn from the lesioning site at a rate of $1 \mathrm{~mm} / \mathrm{min}$. Animals in the Sham-group underwent the same surgical procedures as the 6OHDA group, but were injected only with the vehicle (ascorbic acid solution, at the same concentration mentioned above). All 6OHDA and Sham lesions were placed in the left hemisphere. During the surgery, temperature maintenance was achieved with the use of electrical pads. The surgery was conducted with the aid of a stereotactic frame (Small Animal Stereotaxic Instrument, LS900, David Kopf Instruments, Tujunga, CA, USA) and coordinates were assessed by use of the Paxinos and Watson Atlas (31). Animals were placed in the frame and reference points were defined. In the horizontal plane, the skull point bregma was taken as the reference point. Its position was assessed with the help of an optical microscope. The cortical surface was considered the reference point along the vertical axis, and its position was defined with the aid of electrical means.

Between 21 and 28 days after the lesion procedure, animals were evaluated using the cylinder test (32), which served the purpose of quantifying the asymmetry in motor behavior. The animals were placed in a transparent cylinder for $5 \mathrm{~min}$ and left to explore freely, and only weight supporting touches of the wall were counted, according to the criteria described by Lundblad et al. (33) for the cylinder test. All tests were video-recorded. Animals were killed after the recording-surgery, using a high dose of meperidine, an opioid of choice for euthanasia procedures.

Brains were post-fixed in paraformaldehyde $4 \%$ for $24 \mathrm{~h}$, then dehydrated in ethanol $60 \%$ and embedded in paraffin. Sections with a thickness of $3 \mu \mathrm{m}$ were obtained with a microtome (Leica RM 2235) on Superfrost plus slides, sections were dried over night at $37^{\circ} \mathrm{C}$. Immunohistochemical staining of the substantia nigra pars compacta was performed using tyrosine hydoxylase antibody to investigate dopamine loss (polyconal anti-rabbit, Millipore IHCR1003-6). The sections were de-paraffinized and heat antigen retrieval was performed with citrate buffer pH 6.0 (Dako, Target Retrieval Solution $\mathrm{pH}$ 6.0) for $15 \mathrm{~min}$ in a microwave. Sections were rinsed in PBS and briefly stained with Gill's Hematoxylin, rinsed with water, endogenous peroxidase activity was quenched for $10 \mathrm{~min}$ in $3 \% \mathrm{H}_{2} \mathrm{O}_{2}$ at room temperature. After rinsing with PBS, blocking was performed with Protein-Block (Dako Protein-Block serum free) for $10 \mathrm{~min}$. Primary antibody incubation (non-diluted according to datasheet) was done at room temperature for $60 \mathrm{~min}$, followed by three times wash with PBS. Dako Dual Link (Dako EnVision System HRP, Anti-Mouse/Rabbit Dual Link System) was used as a secondary antibody and incubated for $30 \mathrm{~min}$ at room temperature, followed by a three times PBS wash. Tyrosine-hydroxylase was revealed staining with AECKit HRP Substrate (Invitrogen, ready to use) for $15 \mathrm{~min}$, followed by a PBS rinse. Covers lips were mounted with Kaiser's Glycerin Gelatine (Merck Chemicals). 


\section{ANESTHESIA, ANALGESIA, AND ANTIBIOTIC MEDICATION}

Three complementary drugs were used for achieving anesthesia and analgesia in our study: chloral hydrate, tramadol, and lidocaine. Animals were injected with a $300-\mathrm{mg} / \mathrm{kg}$ intraperitoneal dose of chloral hydrate (at a concentration of $50 \mathrm{mg} / \mathrm{ml}$ ) used as anesthetic. At this dose and concentration of chloral hydrate, a mortality rate of $0 \%$ in adult rats has been reported, while sufficiently deep anesthesia for surgical procedures is achieved (34). Anesthesia can be defined as the concomitant presence of unconsciousness, analgesia, and muscle relaxation (35). In current approaches to anesthesia, these effects are usually obtained with combinations of multiple drugs, since this allows using lower doses and therefore minimizing morbimortality (36). Chloral hydrate is a well-known sedative with potent hypnotic effects, widely used not only in veterinary medicine but also in pediatric and neonatal medicine (37-39). However, it does not have important analgesic effects. In the current protocol, analgesia was achieved with a $4-\mathrm{mg} / \mathrm{kg}$ dose of intraperitoneal tramadol. Tramadol is a drug commonly used in veterinary medicine, which has combined mechanisms of action, a wide safety rank, few side effects and has proven effective for managing surgical pain (4043). Tramadol has been shown to have an analgesic potency similar to meperidine and morphine for treating pain of different origins, including surgical pain $(44,45)$. In our protocol, the tramadol dose was repeated between 12 and $24 \mathrm{~h}$ after the surgery to maintain analgesia, and therefore, it was used both as pre-emptive analgesic and as post-operative medication. Local anesthesia (lidocaine) was used at the incision and at contact points. The eyes of the animals were protected from corneal drying with ophthalmic solution drops. Antibiotic prophylaxis was administered in the form of a single $10 \mathrm{mg} / \mathrm{kg}$ dose of intramuscular cefazoline previous to the surgery.

The choice of the mentioned drug profile responded to particular issues related to the disease model implemented (6OHDAlesion model of PD). Other drug options in laboratory animals include dissociative anesthetics (in particular ketamine and combinations of this drug, for example ketamine-xylazine), barbiturates, and inhalant anesthetics (halothane, isoflurane, sevoflurane, among others). Ketamine-xylazine has been reported to yield neuroprotective effects over the central nervous system (CNS), and it is currently questioned to what degree it interferes with the 6OHDA PD model, which made it unsuitable for the present study (46). In the case of isoflurane, it has been shown to induce apoptosis in the CNS $(47,48)$, which could also have potentially affected the implementation of the 6OHDA-model. Since other inhalant anesthetics have not been analyzed regarding this effect, it is safer to avoid this drug family. Halothane might have been an alternative, but it is a highly hypotensive and arrhythmogenic drug, not necessarily safe in small animals (49).

The anesthetic and analgesic medication used was the same for the lesion- and the neuronal activity recording surgeries, and the stereotactic procedure was repeated as well.

\section{ASSESSMENT OF ANESTHESIA DEPTH}

During the neuronal activity recording surgery, the state of consciousness was characterized periodically (every 10-12 min) by evaluating the tail-pinch reflex with the application of a standardized non-painful stimulus. Methods to assess the state of consciousness under anesthesia in animals have been widely discussed $(50,51)$. We defined the following levels of alertness. Level 1: deep anesthesia, level 2: mild alertness, level 3: full alertness. At level 1, animals did not present a positive paw withdrawal reflex, cutaneous reflex, or tail-pinch reflex. Level 2 was defined as the first time of appearance of a positive tail-pinch reflex, and at level 3 the animals showed a strong response to tail-pinch stimulation, either withdrawing both paws and/or energetically contracting abdominal muscles. Since the evaluation of reflexes is subjective, all the evaluations were conducted by the same person, to avoid inter-personal variation. At the end of the recording surgery, we confirmed the wellness and alertness level of the animals by letting them explore freely through the laboratory.

\section{RECORDING OF NEURONAL ACTIVITY}

After completed motor evaluation, animals went through stereotactic surgery with the objective of registering spontaneous activity of the medial globus pallidus or globus pallidus pars interna (mGP or GPi). This nucleus corresponds to the structure that was previously called entopeduncular nucleus. In the present work, we adhere to the nomenclature proposed by the latest edition of Paxinos and Watson's Atlas and refer to this structure as $\mathrm{mGP}$ or GPi. The time between the lesion and the recording surgery was between 21 and 28 days. This amount of time has been proven to account for a significant level of dopamine-cells and dopamine-content loss in comparison to the contralateral, not-lesioned side of the brain (30). Similar times were allowed between both surgeries for the Sham-group. Following anesthesia, animals were placed in a specially designed restraining device, which was built ad hoc with semi-rigid plastic and covered in the inside with a soft and high quality thermal insulator. The device's purpose was not to keep the animals firmly restrained if they spontaneously moved, as it was only loosely bound. On the contrary, it served the purpose of minimizing discomfort helping animals to stay calm during the surgery. During the whole recording time, the animals did not make any spontaneous movements. If the animals did not relax but, on the contrary, attempted to move during the experiment, we considered that an end-point for the recording-surgery. During the whole surgical procedure, the eyes of the animals were covered and all surgeries were conducted in identical conditions of environmental silence. Following these procedures, we were able to record neuronal activity uninterruptedly during the awakening process and for long periods of time, obtaining up to $3 \mathrm{~h}$ of recording of the same neuron. Recording coordinates fell within the limits defined as the GPi by Paxinos and Watson's Atlas. Neuronal recordings were obtained using glass-insulated platinum/iridium ( $\mathrm{Pt} / \mathrm{Ir}$ 80/20\%) microelectrodes with nominal impedance of $0.8-1.2 \mathrm{M} \Omega$ (mTSPBN-LX1, FHC, Inc., Bowdoin, ME, USA). Signals were amplified, conditioned and monitored with an analog oscilloscope, digitized with a dedicated acquisition system (1401plus, CED) and saved in a PC running Spike 2.0 software. The sampling rate was $20 \mathrm{kHz}$ and total amplification including probe was $\times 10,000$, checked with a built-in calibration signal of $1 \mathrm{mV} \mathrm{p}-\mathrm{p}$ at the beginning of each experiment. 


\section{DATA ANALYSIS}

Signals were processed off-line. Spikes were extracted and classified using the algorithm developed by Quian Quiroga (52). Single units were used to construct interspike intervals (ISI) time series. In order to guarantee stationary conditions, $30 \mathrm{~s}$ of recording following the application of a tail-stimulus were discarded from each time series. To be able to characterize different alertness levels unaffected by the proper transition between them, the time separation between the recording segments analyzed was maximized. For alertness levels 1 and 3, the first and the last segments possible at the beginning and at the end of the recording were selected, respectively. In this way, a time separation of $22.52 \pm 3.79 \mathrm{~min}$ (mean \pm SEM) was obtained between successive recording segments. Although the transition segments were not used for the analysis, the recordings were uninterrupted during the whole awakening process with the objective of guaranteeing that the same neuron was being recorded at different alertness levels.

We quantified the bursting activity by counting the percentage of spikes that triggered a burst (Burst Triggering Spikes, BTS). We applied the rank-surprise algorithm to achieve burst detection (53). Based on this algorithm, we defined the BTS-index as the percentage of bursts over the total number of spikes. As a value limit for the largest ISI to be considered to be part of a burst, we used the p75 of the ISIs distribution. The minimum surprise value accepted was $\alpha=-\log (0.01)$. Entropy was measured using the sample entropy (SE) algorithm developed by Richman and Moorman (54). We analyzed the dependence of the SE with the length of the recording (number of ISIs, $n$ ) and the embedding dimension $(m)$. We chose a tolerance parameter equal to $0.15 \mathrm{SD}$ (SD, standard deviation of the ISIS distribution). The percentage entropy variation for a given alertness transition was calculated as:

$$
\Delta \mathrm{SE}=\frac{\mathrm{SE}_{2}-\mathrm{SE}_{1}}{\mathrm{SE}_{1}} \times 100,
$$

where $\mathrm{SE}_{2}$ is the $\mathrm{SE}$ at a given alertness level and $\mathrm{SE}_{1}$ is the $\mathrm{SE}$ at the previous one. Statistical comparisons between groups were performed using the two-sample $t$-test, and differences were considered significant for $p \leq 0.05$.

\section{RESULTS}

\section{ANIMAL MODEL}

Figure 1 shows the results of the behavioral evaluation through the implementation of the cylinder test for the three animal groups analyzed. We registered a total of $22.17 \pm 1.68$ (mean \pm SEM) touches/test. The left (negative) to right (positive) bias was calculated as the percentage difference between left and right touches over total number of touches. Animals in the 6OHDA group (parkinsonian model) showed a marked asymmetry toward the side ipsilateral to the lesion, reflected by a left bias in the use of the front-limb for weight supporting touches of the wall. On the contrary, both control groups (the Sham- and the not-lesioned groups) showed a right bias. A statistical difference with a $p$-value $<0.01$ was obtained between the 6OHDA-lesioned and both the Sham- and the not-lesioned groups, while no significant difference was observed between the last two. The absence of overlap seen between the 6OHDA-lesioned and both control groups evidenced

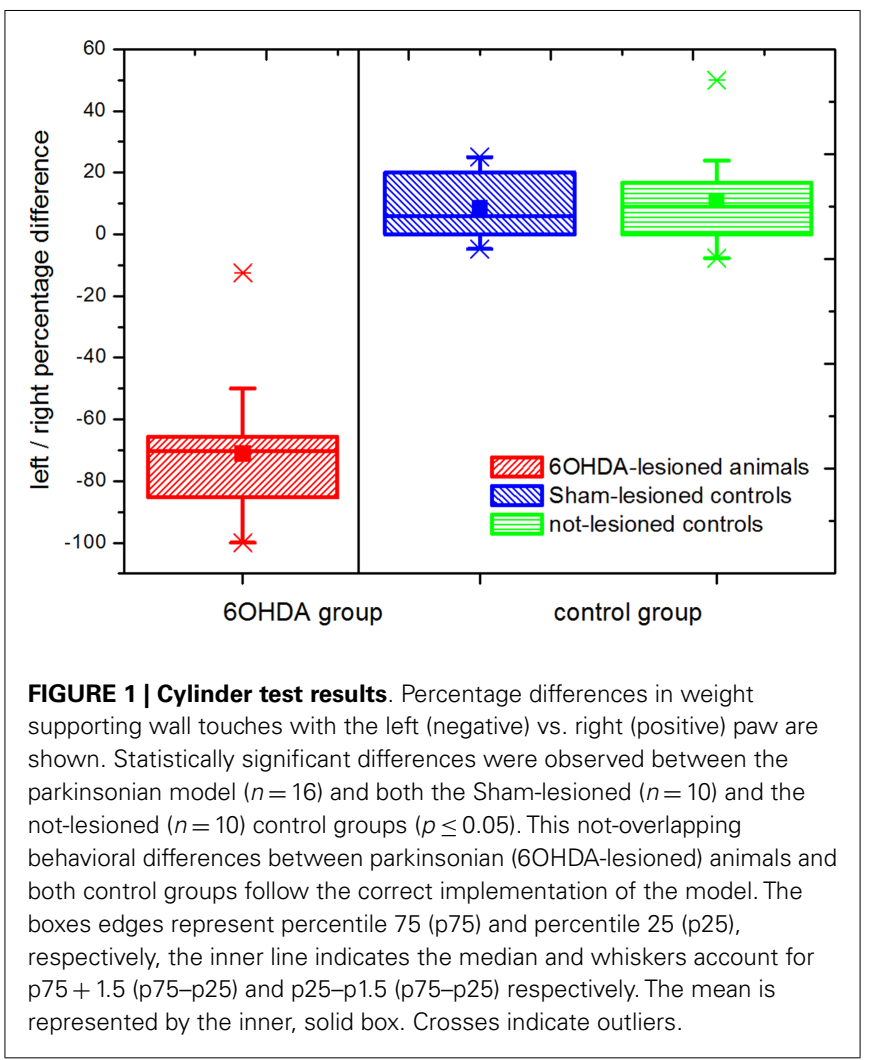

the successful implementation of the 6OHDA- vs. Sham-lesion model.

\section{NEURONAL RECORDINGS}

We obtained a total of 38 recordings, 17 obtained from 166 OHDAlesioned animals and 21 from 20 control animals (10, Sham and 10 , not-lesioned). Eight recordings covered the 3 studied levels of alertness, 13 covered the transition from alertness level 1-2, and 17 covered the transition from alertness level 2-3. For our analysis, 21 recording segments obtained under deep anesthesia (11 controls, 10 parkinsonian), 37 under mild alertness ( 17 controls, 20 parkinsonian), and 24 at full alertness ( 13 controls, 11 parkinsonian) were included. Recording sites can be seen in Figure 2, as determined by stereotactic coordinates. Figure 3 shows sample recording segments of bursting and tonic-firing activity. The mean length time of the recordings was $59.77 \pm 5.07 \mathrm{~min}$, while the segments used for the analysis had a duration of $12.75 \pm 0.72 \mathrm{~min}$ (mean $\pm \mathrm{SEM}$ ). No statistically significant differences were observed between the Sham- and not-lesioned animals for any of the characteristics analyzed in the present work, which will therefore be reported as a single group and referred to as control group.

\section{DISCHARGE RATE AND STATISTICAL PROPERTIES}

Results for statistical properties and BTS-index of the time series are reported in Table 1. Statistical tests were run for comparisons between groups and significant differences are highlighted. The rate of discharge in the control group showed a decreasing trend as alertness increased, whereas in the parkinsonian group an increasing trend was observed (Figure 4). Under these conditions, while 


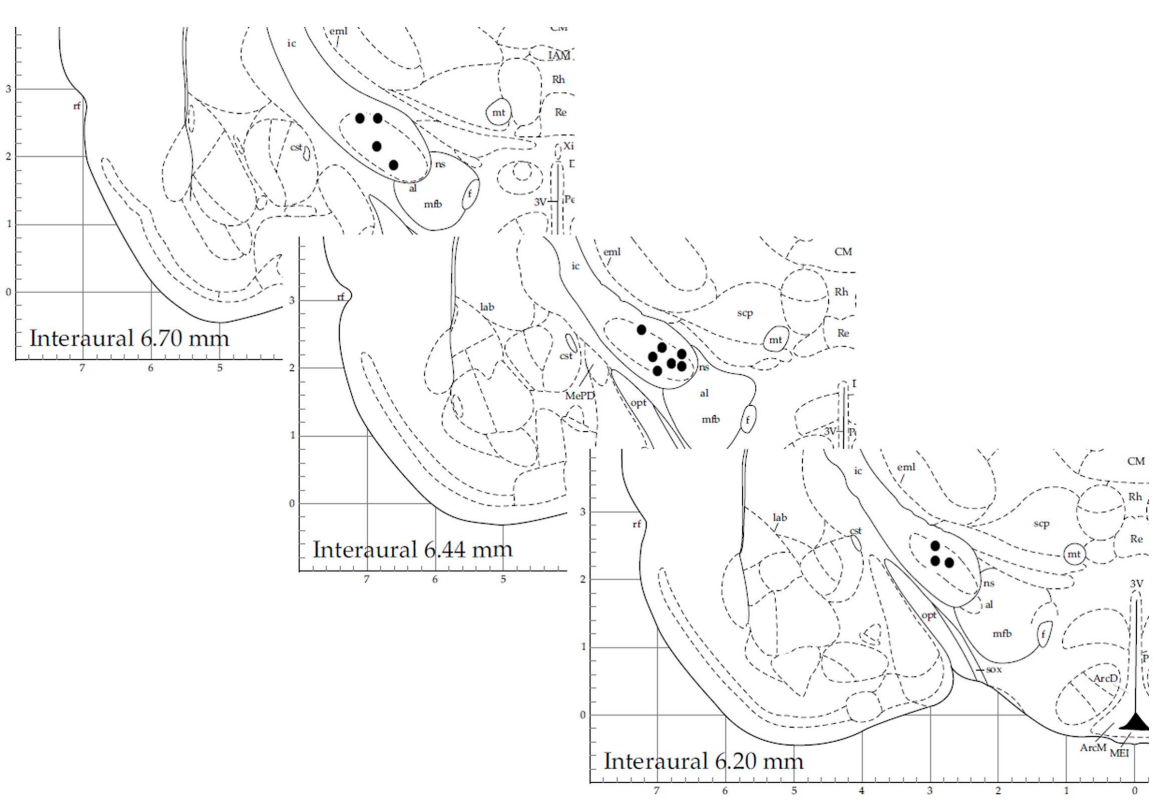

FIGURE 2 | Stereotactic coordinates of recording sites for $\mathbf{1 4}$ sample neurons. This figure is modified from Ref. (31) with permission.
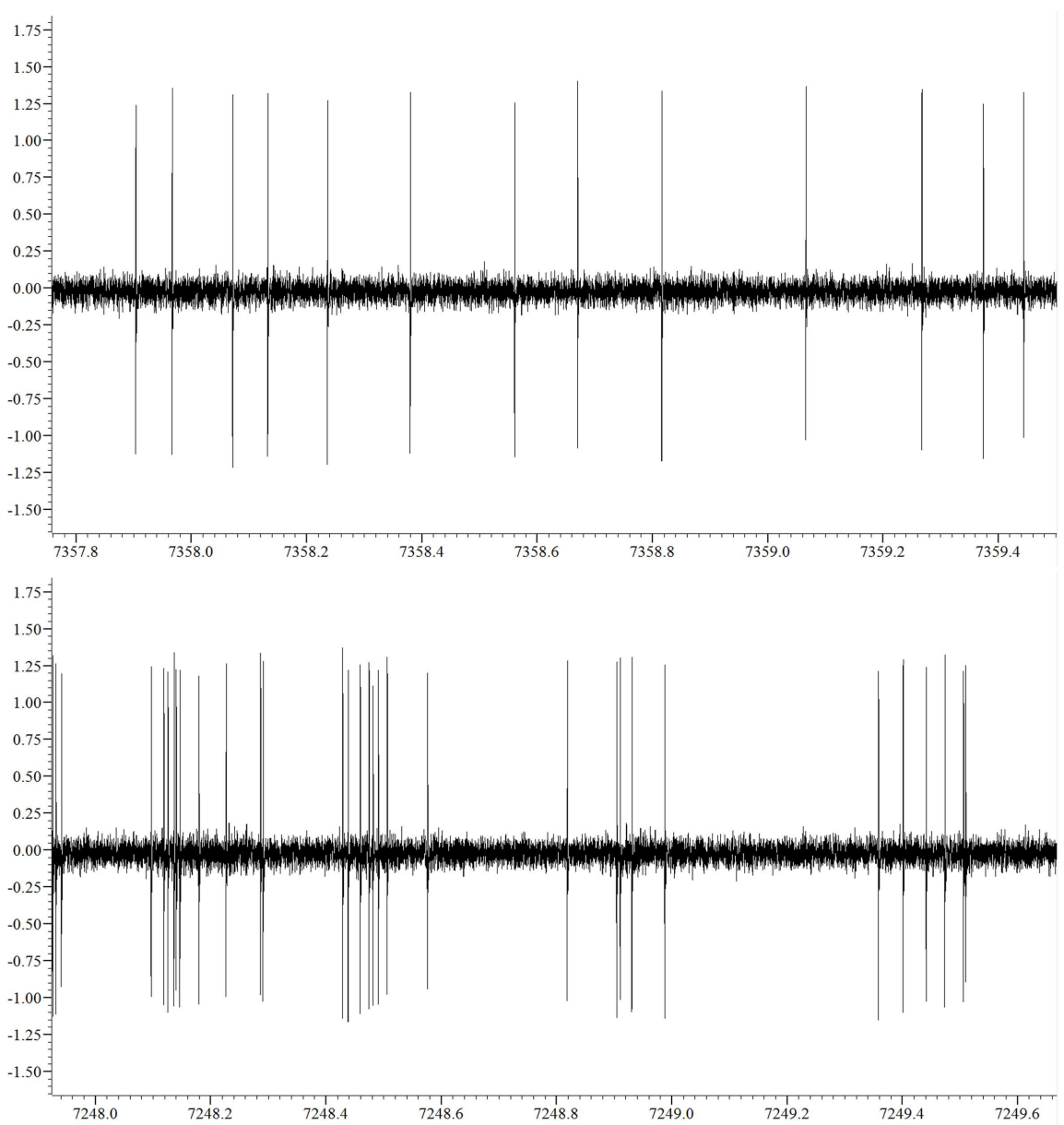

FIGURE 3 | Sample non-bursting (upper panel) and bursting (lower panel) segments of the neuronal activity recorded. 


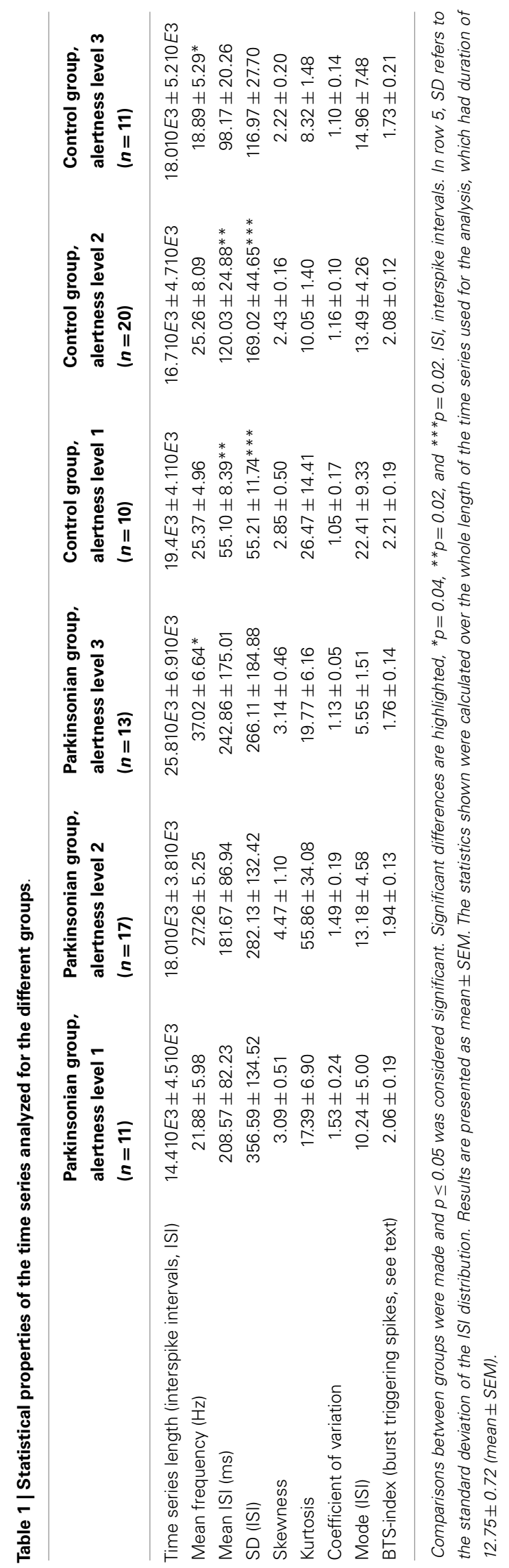

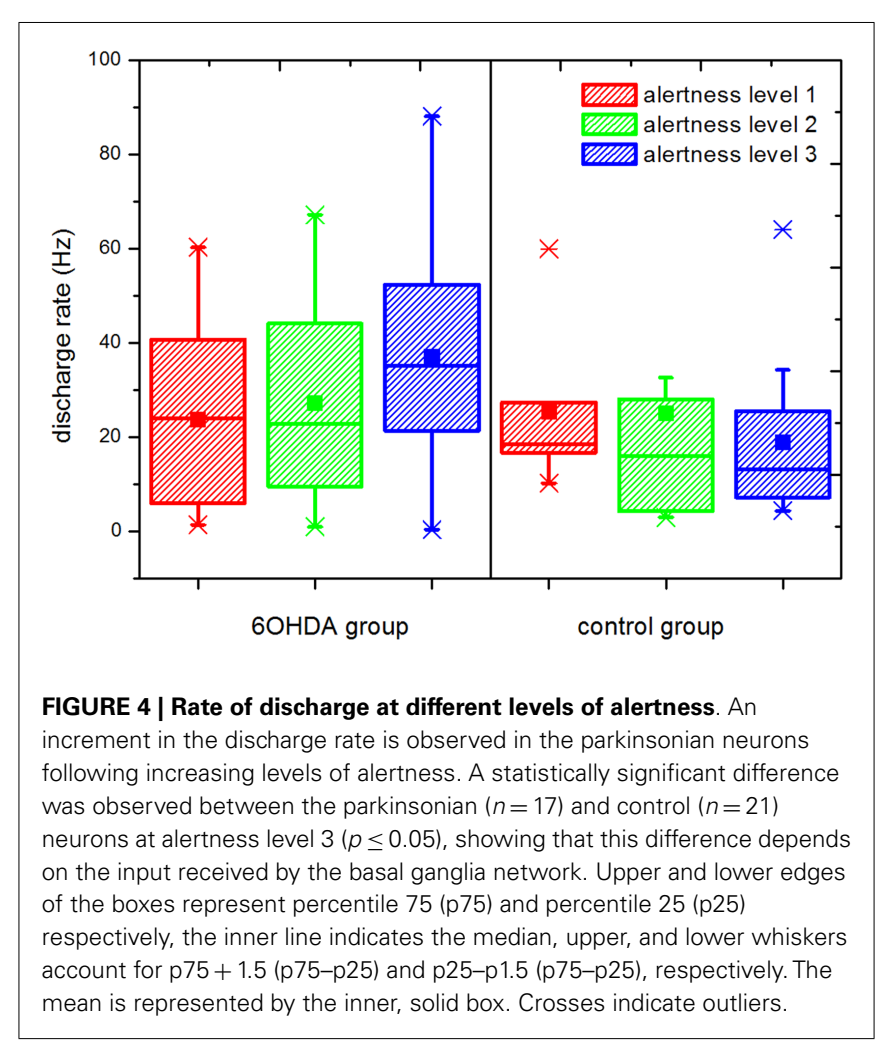

the control group presented a slightly higher discharge rate than the parkinsonian group (not significant) under the effect of anesthesia, the rate was significantly higher for the parkinsonian group in the fully alert state (alertness level $3, p \leq 0.05$ ).

\section{ENTROPY}

We analyzed the dependence of SE with the length of the time series $(n)$ and with the embedding dimension for all the time series included in the study. The value of SE showed asymptotic behavior toward larger $n$. We found a stable value of SE for $n \geq 2500$ for all time series. All the results presented here were obtained with $n=2500$ ISI, and time series shorter than that were excluded. Regarding embedding dimension, SE was calculated for dimensions between 1 and 10 and relative consistency (meaning no changes in the relation between different time series) was found for dimensions between 3 and 5. This analysis was conducted using: first, all the available data points and second, $n=2500$ data points for each time series and differences were not observed. All the results presented here were calculated considering an embedding dimension equal to 5 .

The results for the percentage entropy variation can be seen in Figure 5. With our selection of parameters, a significantly different behavior was observed in the parkinsonian model vs. control groups during the transition from alertness level 2-3 (i.e., from mild to full alertness: transition II). During transition II, an increase in the level of entropy was observed in the parkinsonian neurons, and the percentage of positive variation was higher than during the transition $I$ in the same group (from alertness level $1-2)$. On the contrary, neurons of the control group showed a negligible variation of SE during transition II, even smaller than 


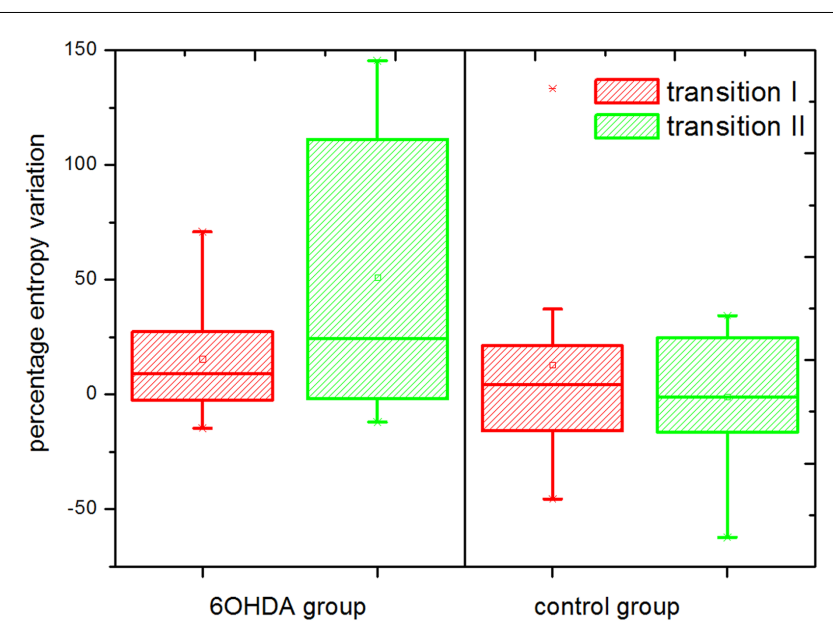

FIGURE 5 | Percentage entropy variation. Transition I and II indicate the percentage variation in the level of sample entropy during the transition from alertness level 1-2, and 2-3, respectively. The entropy variation during transition II was significantly higher in the parkinsonian (6OHDA) than in the control group ( $p \leq 0.05$ ). A number of $n=2500$ data points and an embedding dimension of five were used for the calculation of sample entropy. The boxes edges represent percentile 75 (p75) and percentile 25 (p25), respectively, the inner line indicates the median and whiskers account for $p 75+1.5$ (p75-p25) and p25-p1.5 (p75-p25), respectively. The mean is represented by the inner, solid box. Crosses indicate outliers.

during transition I. The difference in the SE variation between control and parkinsonian neurons was statistically significant at a $95 \%$ confidence level, $p \leq 0.05$. The strength of these results is enhanced by the fact that changes in single neurons across different levels of alertness were recorded, narrowing the effects of population variability.

\section{DISCUSSION}

Our results are in agreement with those found in the literature, where a decrease in the mode and an increase in the skewness, coefficient of variation, and kurtosis were found in alert parkinsonian rats (55). Regarding the frequency of discharge, some works have shown an increment in neurons from the 6OHDArat under anesthesia in comparison to controls, while others did not find statistical differences (56). We did not find statistically significant differences between the groups studied in the rate of discharge under the effect of anesthesia, but as the level of alertness increased the rate of discharge of the neurons in the 6OHDA group was increased as well, while a slight decreasing trend was observed in the control group. A significant difference was found in the fully alert state (alertness level 3) between parkinsonian and control neurons, with a higher discharge rate being observed in the 6OHDA group. The smooth changes observed in the rate of discharge of single cells following increasing levels of alertness highlight the importance of a careful quantification of the anesthesia depth in neurophysiological experiments.

Different methods are available for the calculation of entropy based on time series data $(57,58)$. We used the SE algorithm, which was shown in the literature to be a robust measure for short time series and to demand low computational cost. A positive relation between the level of alertness and the percentage SE variation in the discharge of single neurons in the parkinsonian rat GPi was documented. With a purely statistical approach, it can be shown that the maximum level of entropy of the output nodes of an arbitrarily connected network depends on the level of internal connectivity and the input to the network (59). Experimental evidence in favor of an excessive neuronal connectivity in the parkinsonian BG comes from studies, which showed an increment in the number of inter-neuronal connections in the parkinsonian Str (60). Although these studies haven't been conducted in the GPi yet, it is possible that the number of inter-neuronal connections might be increased also in the GPi and other BG centers in the parkinsonian state. On the other hand, dopamine is known to act modulating the coupling between neurons (61). Therefore, it could also be expected for active mechanisms to be present in the healthy BG reducing the available connections in the alert state, which might offer an explanation for the negligible SE variation during the awakening process in the control group. In the parkinsonian group, our results show clearly that the increase of entropy is manifested during the transition toward the highest level of alertness. This reflects that in $\mathrm{PD}$, the level of entropy in the discharge of single GPi neurons depends on the way the $\mathrm{BG}$ process incoming information. If the increased entropy variation observed is as well related to a pathological amount of inter-neuronal connectivity within the GPi is a question that remains to be elucidated in future works.

Summarizing, our experiments show that the entropy of GPi neurons in vivo varies dynamically depending on the input to the BG network. Therefore, we conclude that entropy needs to be interpreted as a dynamic, emergent property that characterizes the global state of the BG neuronal network, and should not be thought of as a static property of parkinsonian neurons themselves. Regarding the recently proposed entropy hypothesis of movement disorders, this dependence of the GPi neuronal entropy on the global input to the BG network in PD means that with increased BG input (e.g., at full alertness), the communication between the BG and the motor Th/cortex becomes progressively disrupted, which leads to a worsening of akinesia. Since our work was conducted with animals in relaxed, head-restrained condition and devoid of visual or auditory stimuli, it remains for future studies to analyze the relation between the level of entropy at the output centers of the BG, and specific sensory input or motor activity. Finally, the fact that in PD the entropy levels measured from BG neurons at full alertness is not directly comparable with measurements obtained under anesthesia is relevant to the implantation surgery of DBS electrodes, where the use of local vs. general anesthesia is still under discussion (62).

\section{ACKNOWLEDGMENTS}

We would like to thank the technical personnel at the Institute of Neuroinformatics, ETH and UZH, Zürich, Switzerland, and at the laboratories of the Center for Applied Neurological Research, Fleni Institute, Buenos Aires, Argentina, who helped making this work possible.

\section{REFERENCES}

1. Zhou P, Barkhaus PE, Zhang X, Rymer WZ. Characterizing the complexity of spontaneous motor unit patterns of amyotrophic lateral sclerosis using approximate entropy. J Neural Eng (2011) 8:066010. doi:10.1088/1741-2560/8/6/066010 
2. Nemenman I, Bialek W, de Ruyter van Steveninck R. Entropy and information in neural spike trains: progress on the sampling problem. Phys Rev E Stat Nonlin Soft Matter Phys (2004) 69:056111. doi:10.1103/PhysRevE.69.056111

3. Costa M, Goldberger AL, Peng C-K. Multiscale entropy analysis of complex physiologic time series. Phys Rev Lett (2002) 89:068102. doi:10.1103/PhysRevLett. 89.068102

4. Steuer R, Ebeling W, Russell DF, Bahar S, Neiman A, Moss F. Entropy and local uncertainty of data from sensory neurons. Phys Rev E Stat Nonlin Soft Matter Phys (2001) 64:061911. doi:10.1103/PhysRevE.64.061911

5. Darbin O, Adams E, Martino A, Naritoku L, Dees D, Naritoku D. Non-linear dynamics in parkinsonism. Front Neurol (2013) 4:211. doi:10.3389/fneur.2013. 00211

6. Cruz AV, Mallet N, Magill PJ, Brown P, Averbeck BB. Effects of dopamine depletion on network entropy in the external globus pallidus. J Neurophysiol (2009) 102:1092-102. doi:10.1152/jn.00344.2009

7. Darbin O, Soares J, Wichmann T. Nonlinear analysis of discharge patterns in monkey basal ganglia. Brain Res (2006) 1118:84-93. doi:10.1016/j.brainres. 2006.08.027

8. Dorval AD, Russo GS, Hashimoto T, Xu W, Grill WM, Vitek JL. Deep brain stimulation reduces neuronal entropy in the MPTP primate model of Parkinson's disease. J Neurophysiol (2008) 100(5):2807-18. doi:10.1152/jn.90763.2008

9. Lafreniere-Roula M, Darbin O, Hutchison WD, Wichmann T, Lozano AM, Dostrovsky JO. Apomorphine reduces subthalamic neuronal entropy in parkinsonian patients. Exp Neurol (2010) 225:455-8. doi:10.1016/j.expneurol. 2010.07.016

10. Darbin O, Dees D, Martino A, Adams E, Naritoku D. An entropy-based model for basal ganglia dysfunctions in movement disorders. Biomed Res Int (2013) 2013:5. doi:10.1155/2013/742671

11. Sanghera MK, Darbin O, Alam M, Krauss JK, Friehs G, Simpson RK, et al. Entropy measurements in pallidal neurons in dystonia and Parkinson's disease. Mov Disord (2012) 27(S1):S1-523. doi:10.1002/mds.25051

12. Perlmutter JS, Mink JW. Deep brain stimulation. Annu Rev Neurosci (2006) 29:229-57. doi:10.1146/annurev.neuro.29.051605.112824

13. Benabid AL, Pollak P, Gervason C, Hoffmann D, Gao DM, Hommel M, et al. Long-term suppression of tremor by chronic stimulation of the ventral intermediate thalamic nucleus. Lancet (1991) 337:403-6. doi:10.1016/0140-6736(91) 91175- T

14. Blomstedt P, Hariz MI. Deep brain stimulation for movement disorders before DBS for movement disorders. Parkinsonism Relat Disord (2010) 16:429-33. doi:10.1016/j.parkreldis.2010.04.005

15. Poon CC, Irwin MG. Anaesthesia for deep brain stimulation and in patients with implanted neurostimulator devices. Br J Anaesth (2009) 103(2):152-65. doi:10.1093/bja/aep179

16. Machado A, Rezai AR, Kopell BH, Gross RE, Sharan AD, Benabid AL. Deep brain stimulation for Parkinson's disease: surgical technique and perioperative management. Mov Disord (2006) 21(Suppl 14):S247-58. doi:10.1002/mds.20959

17. Lozano A, Hutchison W, Kiss Z, Tasker R, Davis K, Dostrovsky J. Methods for microelectrode-guided posteroventral pallidotomy. Brain (1996) 83:337-50.

18. D’Haese PF, Pallavaram S, Li R, Remple MS, Kao C, Neimat JS, et al. CranialVault and its CRAVE tools: a clinical computer assistance system for deep brain stimulation (DBS) therapy. Med Image Anal (2012) 16:744-53. doi:10.1016/j.media.2010.07.009

19. Guridi J, Rodriguez-Oroz MC, Lozano AM, Moro E, Albanese A, Nuttin B, et al. Targeting the basal ganglia for deep brain stimulation in Parkinson's disease. Neurology (2000) 55(12 Suppl 6):S21-8.

20. Andres DS, Cerquetti DF, Merello M. Finite dimensional structure of the GPi discharge in patients with Parkinson's disease. Int J Neural Syst (2011) 21(3):175-86. doi:10.1142/S0129065711002778

21. Andres DS, Cerquetti DF, Merello M. Turbulence in globus pallidum neurons in patients with Parkinson's disease: exponential decay of the power spectrum. J Neurosc Methods (2011) 197(1):14-20. doi:10.1016/j.jneumeth.2011.01.022

22. Lettieri C, Rinaldo S, Devigili G, Pauletto G, Verriello L, Budai R, et al. Subthalamic nucleus electrophysiological activity in awake and anesthetized patients. Clin Neurophysiol (2012) 123:2406-13. doi:10.1016/j.clinph.2012.04.027

23. Maciver MB, Bronte-Stewart HM, Henderson JM, Jaffe RA, Brock-Utne JG. Human subthalamic neuron spiking exhibits subtle responses to sedatives. Anesthesiology (2011) 115:254-64. doi:10.1097/ALN.0b013e3182217126
24. Duque P, Mateo O, Ruiz F, de Viloria JG, Contreras A, Grandas F. Intraoperative microrecording under general anaesthesia with bispectral analysis monitoring in a case of deep brain stimulation surgery for Parkinson's disease. Eur J Neurol (2008) 15:e76-7. doi:10.1111/j.1468-1331.2008.02166.x

25. Raz A, Eimerl D, Zaidel A, Bergman H, Israel Z. Propofol decreases neuronal population spiking activity in the subthalamic nucleus of parkinsonian patients. Anesth Analg (2010) 111:1285-9. doi:10.1213/ANE.0b013e3181f565f2

26. Okun M, Naim A, Lampl I. The subthreshold relation between cortical local field potential and neuronal firing unveiled by intracellular recordings in awake rats. J Neurosci (2010) 30(12):4440-8. doi:10.1523/JNEUROSCI.5062-09.2010

27. Chaniary KD, Baron MS, Robinson P, Rice AC, Wetzel PA, Shapiro SM. A novel stereotaxic apparatus for neuronal recordings in awake head-restrained rats. J Neurosci Methods (2011) 198:29-35. doi:10.1016/j.jneumeth.2011.02.025

28. Constantinople CM, Bruno RM. Effects and mechanisms of wakefulness on local cortical networks. Neuron (2011) 69(6):1061-8. doi:10.1016/j.neuron. 2011.02.040

29. Available from: http://www.basel-declaration.org

30. Sauer H, Oertel W. Progressive degeneration of nigrostriatal dopamine neurons following intrastriatal terminal lesions with 6-hydroxidopamine: a combined retrograde tracing and immunocytochemical study in the rat. Neuroscience (1994) 59(2):401-15. doi:10.1016/0306-4522(94)90605-X

31. Paxinos G, Watson C. The Rat Brain in Stereotaxic Coordinates. 3rd ed. London: Academic Press (1997).

32. Schallert T, Fleming SM, Leasure JL, Tillerson JL, Bland ST. CNS plasticity and assessment of forelimb sensorimotor outcome in unilateral rat models of stroke cortical ablation parkinsonism and spinal cord injury. Neuropharmacology (2000) 39:777-87. doi:10.1016/S0028-3908(00)00005-8

33. Lundblad M, Andersson M, Winkler C, Kirik D, Wierup N, Cenci MA. Pharmacological validation of behavioural measures of akinesia and dyskinesia in a rat model of Parkinson's disease. Eur J Neurosci (2002) 15:120-32. doi:10.1046/j.0953-816x.2001.01843.x

34. Field KJ, White WJ, Lang M. Anaesthetic effects of chloral hydrate pentobarbitone and urethane in adult male rats. Lab Anim (1993) 27:258-69. doi:10.1258/002367793780745471

35. Stanski DR. Monitoring depth of anesthesia. In: Miller RD, editor. Anesthesia. (Vol. I), New York, NY: Churchill Livingstone (1994), 1127-60.

36. Whelan G, Flecknell PA. The assessment of depth of anaesthesia in animals and man. Lab Anim (1992) 26:153-62. doi:10.1258/002367792780740602

37. Fish R, Danneman PJ, Brown M, Karas A, editors. The Practice of Veterinary Anesthesia: Small Animals, Birds, Fish and Reptiles. Oxford: Elsevier (2008).

38. Buck ML. The use of chloral hydrate in infants and children. Pediatr Pharm (2005) 11(9):1-4.

39. McCarver-May DG, Kang J, Aouthmany M, Elton R, Mowery JL, Slovis TL, et al. Comparison of chloral hydrate and midazolam for sedation of neonates for neuroimaging studies. J Pediatr (1996) 128:573-6. doi:10.1016/S0022-3476(96) 70375-X

40. Lai J, Ma S, Porreca F, Raffa RB. Tramadol, M1 metabolite and enantiomer affinities for cloned human opioid receptors expressed in transfected HN9.10 neuroblastoma cells. Eur J Pharmacol (1996) 316:369-72. doi:10.1016/S00142999(96)00770-4

41. Giusti P, Buriani A, Cima L, Lipartiti M. Effect of acute and chronic tramadol on [3H]-5-HT uptake in rat cortical synaptosomes. Br J Pharmacol (1997) 122:302-6. doi:10.1038/sj.bjp.0701374

42. Cagnardi P, Villa R, Zonca A, Gallo M, Beccaglia M, Luvoni GC, et al. Pharmacokinetics, intraoperative effect and postoperative analgesia of tramadol in cats. Res Vet Sci (2011) 90(3):503-9. doi:10.1016/j.rvsc.2010.07.015

43. Halder S, Bose PK. Post-operative analgesic effect of epidural xylazine in combination with tramadol in dog. Indian J Anim Health (2000) 39(2):51-2.

44. Almeida RM, Escobar A, Maguilnik S. Comparison of analgesia provided by lidocaine, lidocaine-morphine or lidocaine-tramadol delivered epidurally in dogs following orchiectomy. Vet Anaesth Analg (2010) 37:542-9. doi:10.1111/j.14672995.2010.00563.x

45. Lehmann KA. Tramadol for the management of acute pain. Drugs (1994) 47:19-32. doi:10.2165/00003495-199400471-00005

46. Datla KP, Zbarsky V, Dexter DT. Effects of anaesthetics on the loss of nigrostriatal dopaminergic neurons by 6-hydroxydopamine in rats. J Neural Transm (2006) 113:583-91. doi:10.1007/s00702-005-0353-x 
47. Xie Z, Dong Y, Maeda U, Moir R, Inouye SK, Culley DJ, et al. Isoflurane-induced apoptosis: a potential pathogenic link between delirium and dementia. J Gerontol A Biol Sci Med Sci (2006) 61(12):1300-6. doi:10.1093/gerona/61.12.1300

48. Zhang Y, Dong Y, Wu X, Lu Y, Xu Z, Knapp A, et al. The mitochondrial pathway of anesthetic isoflurane-induced apoptosis. J Biol Chem (2010) 285(6):4025-37. doi:10.1074/jbc.M109.065664

49. Rampil IJ, King BS. Volatile anesthetics depress spinal motor neurons. Anesthesiology (1996) 85(1):129-34. doi:10.1097/00000542-199607000-00018

50. Arras M, Autenried P, Rettich A, Spaeni D, Rülicke T. Optimization of intraperitoneal injection anesthesia in mice: drugs dosages adverse effects and anesthesia depth. Comp Med (2001) 51(5):443-56.

51. Heier T, Steen PA. Assessment of anesthesia depth. Acta Anaesthesiol Scand (1996) 40:1087-100. doi:10.1111/j.1399-6576.1996.tb05570.x

52. Quian Quiroga R, Nadasdy Z, Ben-Shaul Y. Unsupervised spike detection and sorting with wavelets and superparamagnetic clustering. Neural Comput (2004) 16:1661-87. doi:10.1162/089976604774201631

53. Gourévitch B, Eggermont JJ. A nonparametric approach for detection of bursts in spike trains. J Neurosci Methods (2007) 160:349-58. doi:10.1016/j.jneumeth. 2006.09.024

54. Richman JS, Moorman JR. Physiological time-series analysis using approximate entropy and sample entropy. Am J Physiol Heart Circ Physiol (2000) 278:H2039-49.

55. Ruskin DN, Bergstrom DA, Walters JR. Nigrostriatal lesion and dopamine agonists affect firing patterns of rodent entopeduncular nucleus neurons. J Neurophysiol (2002) 88:487-96. doi:10.1152/jn.00844.2001

56. Nakanishi H, Kita H, Kitai ST. Intracellular study of rat entopeduncular nucleus neurons in an in vitro slice preparation: electrical membrane properties. Brain Res (1990) 527:81-8. doi:10.1016/0006-8993(90)91063-M

57. Kita H, Kita T. Cortical stimulation evokes abnormal responses in the dopaminedepleted rat basal ganglia. J Neurosci (2011) 31(28):10311-22. doi:10.1523/ JNEUROSCI.0915-11.2011
58. Grassberger P, Procaccia I. Estimation of the Kolmogorov entropy from a chaotic signal. Phys Rev A (1983) 28:2591-3. doi:10.1103/PhysRevA.28. 2591

59. Tanyimboh TT, Templeman AB. Calculating maximum entropy flows in networks. J Oper Res Soc (1993) 44(4):383-96. doi:10.2307/2584416

60. Cepeda C, Walsh JP, Hull CD, Howard SG, Buchwald NA, Levine MS. Dyecoupling in the neostriatum of the rat: I. Modulation by dopamine-depleting lesions. Synapse (1989) 4:229-37. doi:10.1002/syn.890040308

61. Onn SP, Grace AA. Dye-coupling between rat striatal neurons recorded in vivo: compratmental organization and modulation by dopamine. J Neurophysiol (1994) 71(5):1917-34.

62. Benabid AL, Chabardes S, Mitrofanis J, Pollak P. Deep brain stimulation of the subthalamic nucleus for the treatment of Parkinson's disease. Lancet Neurol (2009) 8:67-81. doi:10.1016/S1474-4422(08)70291-6

Conflict of Interest Statement: The authors declare that the research was conducted in the absence of any commercial or financial relationships that could be construed as a potential conflict of interest.

Received: 17 April 2014; accepted: 31 May 2014; published online: 25 June 2014.

Citation: Andres DS, Cerquetti D, Merello M and Stoop R (2014) Neuronal entropy depends on the level of alertness in the parkinsonian globus pallidus in vivo. Front. Neurol. 5:96. doi: 10.3389/fneur.2014.00096

This article was submitted to Movement Disorders, a section of the journal Frontiers in Neurology.

Copyright (c) 2014 Andres, Cerquetti, Merello and Stoop. This is an open-access article distributed under the terms of the Creative Commons Attribution License (CC BY). The use, distribution or reproduction in other forums is permitted, provided the original author(s) or licensor are credited and that the original publication in this journal is cited, in accordance with accepted academic practice. No use, distribution or reproduction is permitted which does not comply with these terms. 